Carnets de géographes

GÉOGRAPHES

15 | 2021

Les dimensions spatiales du maintien de l'ordre

\title{
Géographie de la dispersion des migrations
} subsahariennes au Maroc

Le cas de deux villes-refuge, Tiznit et Taza

Sofia El Arabi

\section{OpenEdition}

Journals

Édition électronique

URL : https://journals.openedition.org/cdg/7545

DOI : $10.4000 /$ cdg. 7545

ISSN : 2107-7266

Éditeur

UMR 245 - CESSMA

Référence électronique

Sofia El Arabi, « Géographie de la dispersion des migrations subsahariennes au Maroc », Carnets de géographes [En ligne], 15 | 2021, mis en ligne le 30 avril 2021, consulté le 27 mai 2021. URL : http:// journals.openedition.org/cdg/7545; DOI : https://doi.org/10.4000/cdg.7545

Ce document a été généré automatiquement le 27 mai 2021.

\section{c) (i) $९$}

La revue Carnets de géographes est mise à disposition selon les termes de la Licence Creative Commons Attribution - Pas d'Utilisation Commerciale - Pas de Modification 4.0 International. 


\section{Géographie de la dispersion des migrations subsahariennes au Maroc}

Le cas de deux villes-refuge, Tiznit et Taza

Sofia El Arabi

\section{Contexte et problématique de la thèse}

1 Cette thèse de Doctorat traite des conséquences de la politique d'externalisation des dispositifs de sécurisation des frontières de l'Union européenne (UE) au Maroc dans le contexte post-crise migratoire de 2015. Le Royaume du Maroc, pays d'émigration est aussi un espace de transit, d'installation et d'attente pour des individus en migration en provenance d'Afrique subsaharienne. Cette recherche est centrée sur l'outil conceptuel de "dispersion" que j'ai introduit en raison de sa fécondité explicative pour appréhender la politique d'immigration et d'asile marocaine en matière de gestion de l'accueil et d'inclusion urbaine des migrants subsahariens dans deux villes moyennes, Tiznit (au Sud) et Taza (au Nord-Est).

2 Sous l'impulsion et l'accompagnement financier de l'UE, le Maroc a ainsi organisé la politique de dispersion afin d'endiguer les mouvements migratoires subsahariens désireux d'atteindre l'Europe par les côtes marocaines ou les enclaves espagnoles de Ceuta et Melilla. À ce titre, la dispersion s'opère via la distribution spatiale et la relocalisation interne des migrants par les autorités publiques. Elle se fait principalement depuis les camps de la frontière nord jusqu'aux villes moyennes de l'intérieur et du Sud marocain. Le processus de «frontiérisation » que j'associe à celui de "dispersion», comme étant l'une de ses dimensions, est devenu l'un des instruments de la gestion de la frontière au Maroc. La problématique de cette thèse interroge ainsi la dispersion comme nouveau mode de contrôle et de pratique des frontières, engendrant une dialectique entre les intérêts étatiques et ceux des migrants dispersés. Elle permet une lecture nouvelle et plus précise du phénomène migratoire 
subsaharien et de sa gestion, marquée à la fois par la contingence des spatialités et temporalités migratoires et les réactions imprévisibles des autorités marocaines. Elle nous éclaire également sur les effets complexes des déplacements forcés en créant de nouvelles villes-étapes, inscrites dans le parcours migratoire, qui posent la question de leur transformation potentielle en « villes-refuges ».

\section{La méthode de recherche adoptée}

3 C'est dans ce contexte général que cette thèse a pour ambition scientifique d'articuler deux dimensions majeures qui apparaissent comme fonctionnant de pair. La première correspond à l'échelle des individus en situation de dispersion. En effet, la politique de frontiérisation engendre un processus de "mise en vulnérabilité » administrative et socio-économique des subsahariens en migration. La seconde rejoint l'échelle des villes moyennes où se déploie un nouvel espace-temps d'accueil des migrants dispersés. Une attention particulière est accordée à la question de l'accueil saisie dans les réalités territorialisées, au plus près des acteurs locaux et des espaces urbains investis par les migrants dispersés. Afin de comprendre cette double dynamique de "mise en vulnérabilité » et de développement d'un accueil, j'ai pris comme unité d'analyse les deux villes moyennes de Taza (région de Fès-Meknès) et Tiznit (région de Souss-Massa). Cette recherche porte la focale sur ces deux villes, de facto assignées à devenir des espaces d'accueil de ces nouveaux arrivants dispersés depuis 2015.

4 Le choix du cadre a été celui d'une recherche-action afin de fonder notre étude empirique tout en l'adossant à une méthode participative, menée comparativement dans les villes moyennes de Tiznit et Taza. À cet effet, j'ai organisé et animé plusieurs groupes de discussion avec le concours de migrants subsahariens dispersés, et la collaboration de la société civile ainsi que des autorités locales des deux villes étudiées. Mes observations et enquêtes réalisées auprès de 215 migrants subsahariens_dont 151 à Tiznit et 64 à Taza entre 2017 et 2019_ ont permis de mettre en lumière les parcours, les motivations et les profils socio-démographiques des migrants dispersés.

5 J'ai mobilisé les critères définis par le Center for Collaborative Research de l'Université de Californie, afin de respecter les normes éthiques de la recherche participative. Cette recherche était orientée vers l'équité, effectuée selon des formats de collaboration et avec le concours de tous les participants. Une première séquence consistait en une collecte d'informations et une co-construction du questionnaire administré aux migrants dispersés. Une deuxième séquence consistait en une enquête individuelle amenant également à des modalités particulières (l'invitation dans un café notamment). Les enquêtés étaient indemnisés pour leur temps à remplir le questionnaire. Dans la méthode de recherche participative, il incombe au chercheur la responsabilité d'établir un climat et un lien de confiance et une indispensable solidarité immédiate vu la précarité des enquêtés. À ce titre, un dispositif scientifique non stigmatisant leur apportant certains bénéfices concrets a été mis en place. Cela s'est traduit par une intégration dans des réseaux d'associations locales, des projets d'ateliers de formation et une organisation de repas collectifs et d'événements caritatifs conduisant à leur mise en contact avec les autorités locales.

D'une part, mes enquêtes ont été construites sur le fondement du principe de convivialité qui se traduit par une écoute attentive des récits de vie des migrants et le choix d'un cadre adapté agréable (un local associatif, une salle de café) où les enquêtés 
sont invités autour d'une collation. D'autre part, le principe de négociation collective a été privilégié notamment au sujet de l'indemnité des migrants et l'intérêt porté à leur condition spécifique et non à des trajectoires emblématiques. Parallèlement, je me suis investie à titre personnel dans l'accueil des migrants au sein des deux villes à travers la co-fondation de l'association ASAM (Association de Solidarité pour l'Appui des Migrants) à Taza en 2017 ainsi que dans l'organisation d'événements caritatifs. J'ai pu ainsi apprécier la nécessité de la démarcation entre l'approche du chercheur et celle de l'engagement citoyen et humanitaire.

\section{Principaux résultats}

7 Un premier résultat a été de constater que la dispersion vise aussi bien les migrants en situation irrégulière que des personnes régularisées. Ensuite, des catégories associées à des caractéristiques de vulnérabilité_ les femmes enceintes, les mineurs non accompagnés ou les réfugiés_ ont été ciblées par la politique de dispersion et comparées avec les dispositions de la loi 02-03 relative à l'entrée et au séjour des étrangers au Maroc, à l'émigration et l'immigration irrégulières. La dispersion telle qu'elle se déploie au Maroc (voir figure 1 ci-dessous) vise à atténuer voire prévenir la concentration spatiale des migrants, leur regroupement en un lieu et en une masse, et ainsi réduire la pression sur les points de passage en Europe telle la ville de Tanger, sise à l'extrémité du nord-ouest du pays sur le détroit de Gibraltar.

Figure 1. Le phénomène de dispersion des migrants subsahariens de la frontière nord-marocaine vers les villes intérieures et le Sud marocain

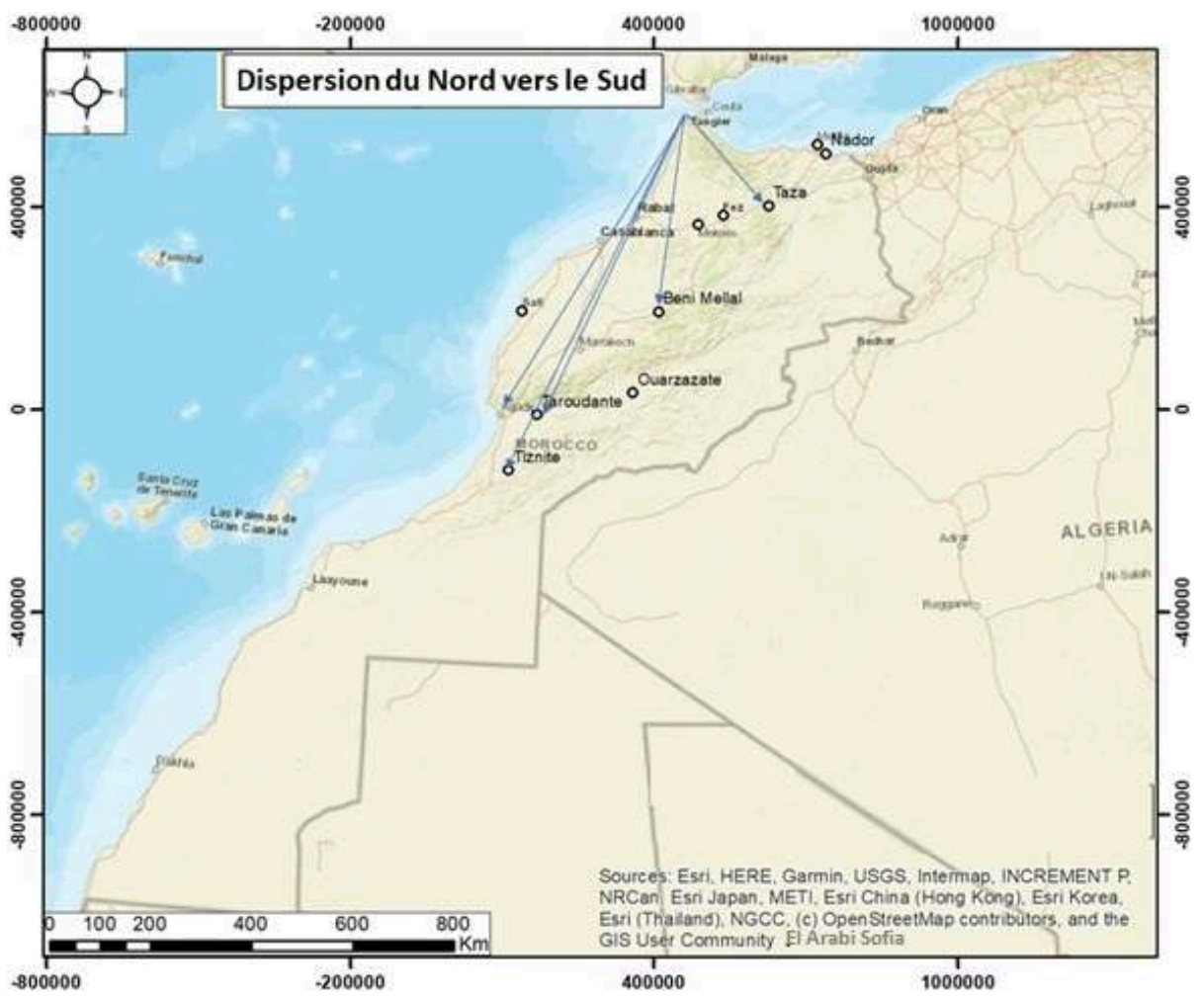

Source : Sofia El Arabi, carte réalisée à partir de données de terrain collectées entre 2017 et 2020 à Tiznit et Taza. 

termes dynamiques de la réalité de la dispersion, ce qui permet de mieux rendre compte de la complexité du parcours migratoire dans sa macro et sa micro-réalité. La dispersion transcende le simple acte de déplacement physique contraint (organisé ou fortuit). Mes investigations sur le terrain ont permis d'élargir le concept de dispersion à d'autres dimensions (entre autres axiologiques et éthiques) que celles se référant plus classiquement aux politiques publiques. La relation apparente avec d'autres enjeux majeurs, telles les nouvelles stratégies d'ancrage urbain des individus dispersés et surtout les velléités locales d'accueil, m'ont conduite à repenser les effets de la dispersion dans sa durée.

Cette recherche a également révélé, en même temps que confirmé, une politique de "frontiérisation" (Mbembe, 2020) se traduisant par un effet d'épaississement de la frontière maroco-européenne. Dans cette optique, la thèse de recherche met en exergue les divergences d'actions publiques territorialisées entre lieux de contrôle (parfois d'expulsion) et lieux d'accueil (villes-refuge). La dispersion se phénoménalise tel un continuum frontalier opposant la violence dans les espaces frontaliers du Nord où s'expriment les «faits de frontière » (Schmoll, 2020) à l'accueil au sein des villes moyennes. Elle redéfinit ainsi la notion de frontière et l'installe dans une dimension concrète de centaines de kilomètres. La frontière devient flexible et se dilate d'autant qu'elle est nourrie par le transfrontiérisme.

10 La thèse interroge par ailleurs la frontière en tant qu'expérience de «mise en vulnérabilité » des subsahariens dispersés et « d'écartèlement » du passage converti en séjour de transit fragmenté. Les migrants dispersés adoptent une forme de mobilité circulaire, instaurent un système d'aller-retour en demeurant en interconnexion continue avec les villes frontalières du Nord et les villes étudiées. À cet égard, un changement d'échelle s'impose pour la reconstitution de ces parcours migratoires dispersés (re) composés de lieux hospitaliers et de lieux hostiles. Mon travail de recherche mené in situ dans deux villes-refuges montre comment ce changement d'échelle peut être générateur d'un horizon de possibilités dont celle de la ville-refuge comme contrepoint inattendu aux politiques publiques coercitives.

11 La condition de vulnérabilité des migrants enquêtés a engendré des dynamiques de solidarité et se voit donc amortie par les sociétés civiles localisées dans les «villesrefuges » sous le sceau d'éthiques de l'hospitalité. Il est alors possible d'interroger les stratégies d'une cohabitation urbaine dans l'apparition d'un vécu nouveau de la frontière des migrants dont la visibilité est associée à leur vulnérabilité consubstantielle du fait même de leur dispersion. Cette dernière les fige dans des pratiques de mendicité en tant que source principale de survie immédiate. Ils posent de ce fait des questions de bienfaisance, d'inclusion urbaine et d'hospitalité. La ville-refuge devient ainsi un espace de résistance à la construction de la frontière, s'affirme comme espace «de l'être et de l'agir ensemble» (Di Méo, 2005) afin d'assurer un équilibre fragile entre deux présences. À l'échelle de ces villes, de nouveaux engagements émergent avec l'implication des pouvoirs locaux affichant une forme de bienveillance et d'adhésion à des valeurs positives de l'accueil. En définitive, la ville-refuge comporte concomitamment la promesse et le liseré d'un "droit à la vie urbaine », une forme d'inclusion et de coexistence sociales (Lefebvre, 1968) fondées sur des capacités hospitalières plurielles. Celles-ci établissent les fondements d'une politique de « l'encommun » (Mbembe, 2020).

Carnets de géographes, 15 | 2021 
12 J'ai pu observer le devenir du migrant et sa constante recherche d'équilibre entre l'expérience de la dispersion et le développement de tactiques d'ajustement. Afin d'en rendre raison, j'ai été amenée à forger et à introduire le concept de «civilité de transit». Cela signifie que le migrant agit sur les conséquences factuelles de la dispersion en tentant de paralyser les frontières de l'hostilité érigées entre lui-même et les autochtones. La « civilité de transit » se traduit par des stratégies adaptatives et une mise en conformité de leur présence en ville avec les singularités socio-culturelles locales. Elle est ordonnée par la nécessité de préserver un modus vivendi maintenant ouvert «le flux des interactions» (Goffman, 1988) entre migrants-acteurs et autochtones tout en offrant la possibilité de prolonger le séjour en ville. Une cartographie fine des espaces investis par les migrants dispersés a été produite à cette fin et montre qu'ils ne sont pas soumis à un impératif d'invisibilité et peuvent accéder à différentes ressources urbaines en produisant cette "civilité de transit». Elle permet ainsi l'identification de micro-ressources pour rendre l'étape endurable au point qu'elle puisse devenir un espace ponctuel de refuge dans un moment d'attente prolongée et éclatée.

\title{
En guise de conclusion
}

L'examen de la géographie de la dispersion des migrations subsahariennes met en évidence la logique de "frontiérisation». Celle-ci alterne à la fois le registre du sécuritaire et le registre humanitaire et résonne sous forme d'arrangements instables. La dispersion impose une logique de court terme qui se perpétue et produit un continuum de vulnérabilité. Le migrant dispersé apparaît finalement, à travers cette recherche, comme un acteur engagé dans « une lutte pour la reconnaissance sociale » (Honneth, 2013). Il (re) négocie et reconfigure de manière précaire sa présence dans la ville-refuge où l'hospitalité relève d'une approche déontologique tout en étant envisagée sous l'angle humanitaire et compassionnel de la situation d'urgence. Convoquant à la fois la géographie, la politique et l'éthique, la dispersion interroge notre capacité à reconnaître le migrant dans la singularité de ses aspirations. Le chercheur est ainsi invité à repenser les enjeux de coexistence urbaine dans leur dimension d'agir et d'être-ensemble et l'exigence d'une mise en place de réelles politiques publiques concertées face aux enjeux des migrations du xxi ${ }^{e}$ siècle.

\author{
Discipline \\ Géographie politique, culturelle et historique

\section{Directrice} \\ Sylvie Brunel, Professeure des universités, Sorbonne Université

\section{Université} \\ Faculté des Lettres de Sorbonne Université \\ Composition du jury de thèse, soutenue le 09 décembre 2020 : \\ Sylvie Brunel, Professeure des universités, Sorbonne Université (directrice de \\ thèse) \\ Catherine Fournet-Guerin, Professeure des universités, Sorbonne Université \\ (présidente du jury)
}


Catherine Wihtol De Wenden, Directrice de recherche émérite au CNRS et Enseignante-chercheuse, Sciences Po-Paris (rapporteure)

Mehdi Alioua, Professeur associé en Sociologie et Directeur pédagogique de Sciences Po Rabat, Université Internationale de Rabat (rapporteur)

David Goeury, Docteur en géographie et Enseignant-chercheur, Agence de l'enseignement français à l'étranger, Lycée Descartes de Rabat (co-encadrant)

\section{Situation professionnelle au moment de la publication}

Docteure en géographie politique, culturelle et historique de l'Université Sorbonne Université, École doctorale de géographie de Paris (ED 434)

Enseignante vacataire à l'Université Paris-Diderot, Université de Paris assurant l'enseignement « Métiers des géographes et projet étudiant » dans le cadre de la licence 2 - Domaine Sciences humaines et sociales, mention Géographie et Aménagement.

Courriel : sofiaelarabisorbonne [at] gmail.com

\section{BIBLIOGRAPHIE}

Deschamps C., Lae J-F., Overney L., Proth B. (2018), Parcours de l'exilé : du refuge à l'installation. Programme de recherche "L'accueil, la circulation et l'installation des migrants ", Plan Urbanisme Construction Architecture (PUCA), Ministère de la Transition écologique et solidaire/Ministère de la Cohésion des territoires.

Di Meo G., Buleon P. (2005), Quelle place pour l'individu en géographie sociale ? Lecture géographique des sociétés, Paris, Armand Colin.

Fournet-Guerin C. (2017), L'Afrique cosmopolite. Circulations internationales et sociabilités citadines, Rennes, Presses universitaires de Rennes.

Goffman E. (1988), Les Moments et leurs hommes, Textes recueillis et présentés par Yves Winkin, Paris, Seuil.

Honneth A. (2013), La lutte pour la reconnaissance, Paris, Gallimard.

Lefebvre H. (1968), Le droit à la ville, Paris, Anthropos.

Mbembe A. (2020), Brutalisme, Paris, La Découverte.

Stavo-Debauge J. (2017), Qu'est-ce que l'hospitalité ? Recevoir l'étranger à la communauté, Montréal, Liber.

Schmoll C. (2020), Les damnées de la mer. Femmes et frontières en Méditerranée, Paris, La Découverte.

\section{INDEX}

Thèmes : Carnets de soutenances 
AUTEUR

SOFIA EL ARABI

Docteure en géographie, Enseignante vacataire à l'Université Paris-Diderot.

sofiaelarabisorbonne[at]gmail.com 